\title{
Automatic sleep staging using ear-EEG
}

\author{
Kaare B. Mikkelsen ${ }^{1 *}\left(\mathbb{D}\right.$, David Bové Villadsen ${ }^{1}$, Marit Otto ${ }^{2}$ and Preben Kidmose ${ }^{1}$
}

\author{
${ }^{*}$ Correspondence: \\ mikkelsen.kaare@eng.au.dk \\ 1 Department \\ of Engineering, Aarhus \\ University, Finlandsgade 22, \\ 8200 Aarhus N, Denmark \\ Full list of author information \\ is available at the end of the \\ article
}

\begin{abstract}
Background: Sleep and sleep quality assessment by means of sleep stage analysis is important for both scientific and clinical applications. Unfortunately, the presently preferred method, polysomnography (PSG), requires considerable expert assistance and significantly affects the sleep of the person under observation. A reliable, accurate and mobile alternative to the PSG would make sleep information much more readily available in a wide range of medical circumstances.
\end{abstract}

New method: Using an already proven method, ear-EEG, in which electrodes are placed inside the concha and ear canal, we measure cerebral activity and automatically score the sleep into up to five stages. These results are compared to manual scoring by trained clinicians, based on a simultaneously recorded PSG.

Results: The correspondence between manually scored sleep, based on the PSG, and the automatic labelling, based on ear-EEG data, was evaluated using Cohen's kappa coefficient. Kappa values are in the range 0.5-0.8, making ear-EEG relevant for both scientific and clinical applications. Furthermore, a sleep-wake classifier with leave-one-out cross validation yielded specificity of 0.94 and sensitivity of 0.52 for the sleep stage.

Comparison with existing method(s): Ear-EEG based scoring has clear advantages when compared to both the PSG and other mobile solutions, such as actigraphs. It is far more mobile, and potentially cheaper than the PSG, and the information on sleep stages is far superior to a wrist-based actigraph, or other devices based solely on body movement.

Conclusions: This study shows that ear-EEG recordings carry information about sleep stages, and indicates that automatic sleep staging based on ear-EEG can classify sleep stages with a level of accuracy that makes it relevant for both scientific and clinical sleep assessment.

Keywords: EEG, Ear-EEG, Mobile EEG, Sleep scoring

\section{Background}

Sleep [1] and the quality of sleep has a decisive influence on general health [2-4], and sleep deprivation is known to have a negative impact on overall feeling of well-being, and on cognitive performance such as attention and memory [5]. However, sleep quality is difficult to measure, and the current gold standard, polysomnography (PSG) [6] requires expert assistance and expensive equipment. Moreover, characterizing sleep by means of conventional PSG equipment will inevitably have a negative impact on the sleep, and thereby bias the sleep quality assessment. Because of the need for professional 
assistance in PSG acquisition, and because of the laborious process to evaluate PSG data, sleep assessment is in most cases limited to a single or a few nights of sleep.

Due to these circumstances, there is an ongoing effort to explore other options for high-quality sleep monitoring [7, 8]. A very promising candidate in this field is ear-EEG [9], due to its potential portability and the fact that it conveys much of the same information as the PSG, namely EEG data [10]. It is likely that the ear-EEG technology will have a much lower impact on the quality of sleep, giving a more accurate picture of the sleep, and also be suitable for sleep assessment over longer periods of time. Recently, the feasibility of ear-EEG for sleep assessment has been studied in a few exploratory papers [11-13], all indicating that ear-EEG is a very promising candidate.

This paper is based on a new dataset comprising nine healthy subjects recorded simultaneously with both PSG and ear-EEG for one night. This is significantly more sleep data than in previous studies. Trained clinicians manually scored the sleep following the guidelines of the American Academy of Sleep Science (AASM) [14]. The sleep staging based on ear-EEG was based on an automatic sleep staging approach, where a statistical classifier was trained based on the labels from the manual scoring (for other examples of this, see [15-17]). The automatic sleep staging was chosen for two reasons: (i) there was not any established methodology for sleep staging based on ear-EEG, while the machine learning approach provided rigorous and unbiased sleep staging. (ii) The question of whether a given method can also be used without manual scoring is important whenever wearable devices for long term monitoring are discussed.

In the "Results" section below, additional support for this reasoning is presented, based on waveforms.

\section{Methods}

\section{Research subjects}

For this study, nine healthy subjects were recruited, aged 26-44, of which three were female. Measurements were all conducted in the same way: subjects first had a partial PSG [consisting of six channel EEG, electrooculography (EOG) and electromyography (EMG) on the chin] mounted by a professional at a local sleep clinic. Subsequently the subject was transported to our laboratory where the ear-EEG was mounted.

The subjects went home and slept with the equipment (both PSG and ear-EEG) for the night, and removed it themselves in the morning. The subjects were instructed to keep a cursory diary of the night, detailing comfort and whether the ear-EEG ear plugs stayed in during the night.

\section{EEG hardware}

The ear plugs used in this study were shaped very similarly to those used in [18], with the difference that the plugs here were made from soft silicone, and the electrodes were solid silver buttons soldered to copper wires. See Fig. 1 for an example of a left-ear plug. Before insertion, the outer ears were cleaned using skin preparation gel (NuPrep, Weaver and Company, USA) and electrode gel (Ten20, Weaver and Company, USA) was applied to the electrodes. Ear-EEG electrodes were ELA, ELB, ELE, ELI, ELG, ELK, ERA, ERB, ERE, ERI, ERG, ERK, as defined in [19]. 

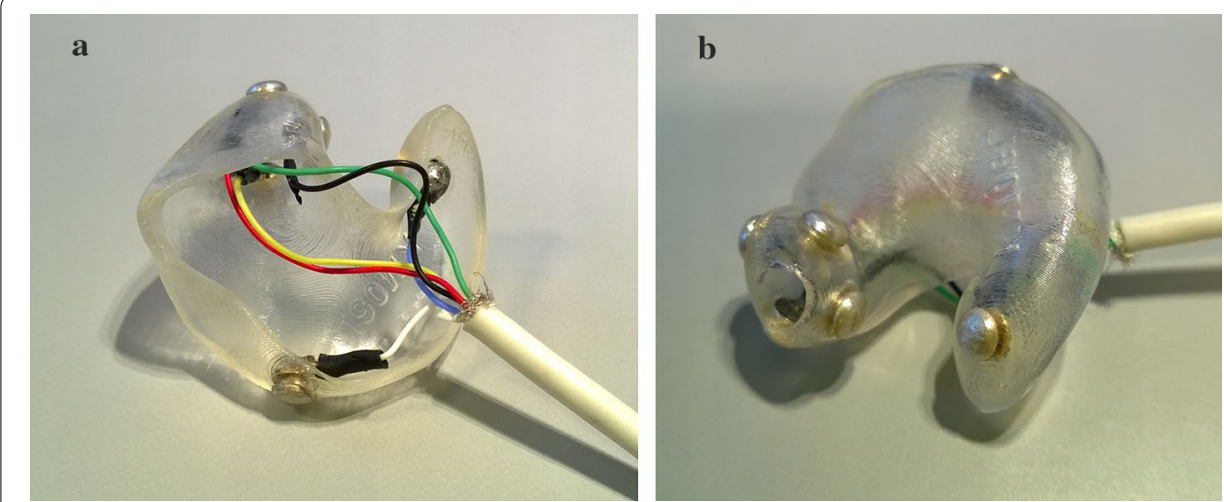

Fig. 1 Example left-ear ear plug with silver electrodes.

As described in [18], ear-EEG electrodes were validated by measuring the auditory steady state responses (ASSR) using $40 \mathrm{~Hz}$ amplitude modulated white noise, which was performed while the subject was still in the laboratory. All electrodes (including ear-EEG) were connected to the same amplifier (Natus xltek, Natus Medical Incorporated, USA), and ear-EEG electrodes were Cz-referenced during the recording. The PSG consisted of two EOG electrodes, two chin EMG electrodes and 8 scalp electrodes (O1, $\mathrm{O} 2, \mathrm{C} 3, \mathrm{C} 4, \mathrm{~A} 1, \mathrm{~A} 2, \mathrm{~F} 3, \mathrm{~F} 4$ by the 10-20 naming convention). The data was sampled at $200 \mathrm{~Hz}$.

\section{Sleep scoring}

\section{Manual scoring}

All PSG-measurements were scored by trained experts at the local sleep clinic, according to the AASM guidelines [14]. The scorers did not use the ear-EEG data in any way, and did not receive any special instructions regarding this data. Scoring was done based on 30-s non-overlapping epochs, such that each epoch was assigned a label from the set: W, REM, NREM1, NREM2, NREM3. We direct the reader to the established sleep literature (such as [14]) for a discussion of these labels.

\section{Automatic scoring}

To investigate the hypothesis that ear-EEG data can be used for sleep scoring, machine learning was used to train an automatic classifier to mimic the scoring of the sleep experts. The analysis pipe line used for this is described below.

\section{Channel rejection}

Even though the ear-EEG electrodes were qualified in the lab by measuring an ASSR, it was found in the analysis of the sleep EEG, that some of the ear-EEG channels were noisy. This was probably due to a deterioration in the electrode-body contact from the time when the subject left the lab until they went to bed. The deterioration may also be related to deformation of the ear, when the subject laid their head on the pillow. Because of this deterioration, it was necessary to perform a channel rejection prior to the analysis of the data. This was done in the following way: 
All intra-ear derivations were calculated, and the power in the $10-35 \mathrm{~Hz}$ frequency band was calculated. If $p_{i j}$ is the power calculated for the derivation consisting of channels $i$ and $j$, let $m_{i}=\operatorname{median}\left(\left\{p_{i j}\right\}_{j}\right)$. Electrode $i$ was then rejected if $m_{i}>5 \cdot 10^{-12} \mathrm{~V}^{2} / \mathrm{Hz}$. This uses the fact that a high-impedance electrode will tend to have much more high-frequency noise, and that this will be the case for all derivations that it takes part in. Elegantly, it does not require a simultaneous 'ground truth' electrode, such as a scalp measurement, to determine good and bad electrodes. The value of $5 \cdot 10^{-12} \mathrm{~V}^{2} / \mathrm{Hz}$ was determined by observing which value cleanly separated the electrodes into two groups, commensurate with the knowledge from the ASSR measurements and the subject diaries (for instance, one subject reported having removed one ear plug entirely before falling asleep). See Appendix A for a visualization of this separation. In total, 14 electrodes were rejected out of a possible 72 , resulting in a rejection rate of $19 \%$.

We note that the band-pass filtering of 10-35 Hz was only chosen and performed for the sake of this channel rejection. The non-filtered data set was passed to the next stage of the analysis, as described below.

\section{Feature extraction}

The eight ear-EEG channels were distilled into three derivations $(\langle\cdot\rangle$ denotes average):

L-R: $\langle E L A, E L B, E L E, E L I, E L G, E L K\rangle$

$$
\text { - }\langle E R A, E R B, E R E, E R I, E R G, E R K\rangle
$$

L: $\langle E L A, E L B\rangle-\langle E L E, E L I, E L G, E L K\rangle$

R: $\langle E R A, E R B\rangle-\langle E R E, E R I, E R G, E R K\rangle$

Note that the $\mathrm{L}$ and R-channels describe the potential differences between concha and channel electrodes in each ear. If an electrode was marked as bad, it was excluded from the averages. If this meant that one of the derivations could not be calculated (for instance, if both ELA and ELB were missing), that derivation was substituted with a copy of one of the others. This was only done in the case of subject 5 , which was missing data from the right ear plug.

When choosing features, we were inspired by [15], and chose the list of features shown in Table 1. Of these, a subset were not used by [15] and are described in Appendix B. In general, the time and frequency domain features were based on a 2-32 Hz-bandpass filtered signal, while the passbands for EOG and EMG features were $0.5-30$ and 32-80 Hz, respectively. A $50 \mathrm{~Hz}$ notch filter was also applied. All frequency domain features were based on power spectrum estimates using Welch's algorithm with segment length $2 \mathrm{~s}, 1 \mathrm{~s}$ overlap and applying a Hanning window on each window.

It is important to stress that the EOG and EMG proxy features discussed in this paper were extracted entirely from ear-EEG data-no EOG or EMG electrodes were used in the analysis. This was to distill as much information about EOG and EMG variation as possible from the ear-EEG data.

All 33 features were calculated for each of the three derivations. As described in Appendix B, an attempt was made to reduce the number of features. However, this did not yield satisfactory results, and instead all 99 features were used in the study. 
Table 1 Features used in this study

\begin{tabular}{lll}
\hline Label & Short description & Type \\
\hline F1 & Signal skewness & EEG time domain \\
F2 & Signal kurtosis & \\
F3 & Zero crossing rate & \\
F4 & Hjorth mobility & \\
F5 & Hjorth complexity & \\
F6 & 75th percentile & \\
F7 & Channel correlation & \\
F8 & EMG power & \\
F9 & Minimal EMG power & \\
F10 & Relative EMG burst amplitude & \\
F11 & Slow eye movement power & \\
F12 & Rapid eye movement power \\
F13, F14, F15, F16 & Relative power in $\alpha, \beta, \theta, \delta$-bands & \\
F17, F18, F19, F20, F21, F22 & Power-ratios: $\alpha / \delta, \delta / \beta, \delta / \theta, \theta / \alpha, \theta / \beta, \alpha / \beta$ & \\
F23 & $(\theta+\delta) /(\alpha+\beta)$ & \\
F24 & Spectral edge frequency domain \\
F25 & Median power frequency & \\
F26 & Mean spectral edge frequency difference & \\
F27 & Peak power frequency & \\
F28 & Spectral entropy & \\
F29 & Spindle probability & \\
F30 & Frequency stationarity & \\
F31 & Lowest adj. frequency similarity & \\
F32 & Largest CWT value & \\
F33 & Longest sleep spindle & \\
\hline F16 proxies & \\
& & \\
& &
\end{tabular}

F1-6 and F13-25, 27, 28 are copied from [15], see Appendices A, B for a precise mapping between these features and those in [15]

\section{Classifier training}

\section{Type of classifier}

We used ensembles of decision trees, called a 'random forest' [20], with each ensemble consisting of 100 trees. The implementation was that of the 'fitensemble1' function in Matlab 2015b, using the 'Bag' algorithm. This means that each decision tree is trained on a resampling of the original training set with the same number of elements (but with duplicates allowed), and each tree has a minimum leaf size of 1 . For each tree, splitting is done such that the Gini coefficient [21] is optimized, and continues until all leaves (subgroups) are either homogeneous or have the minimum leaf size.

\section{Cross validation}

We explored three different ways to select test and training data for the classifier (described graphically in Fig. 2):

Leave-one-out Data was partitioned into nine subsets, each subset corresponding to a single subject. Thus the classifier had not seen any data from the person which it was tested on. 


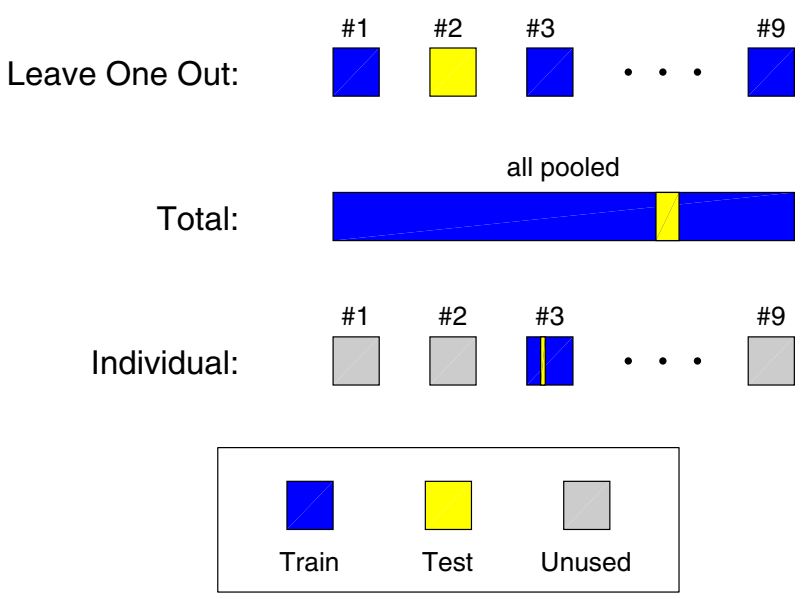

Fig. 2 Graphical illustration of the three cross validation methods. The 'Leave-one-out' method use all epochs from eight subjects for training, and all epochs from the remaining subject for testing. This is done for all nine subjects (ninefold cross validation). In the 'Total' method, all epochs from all subjects are pooled together and partitioned into 20 subsets. The classifier is trained on 19 subsets and tested on one subset. This is done for all 20 combinations of subsets (20-fold cross validation). In the 'Individual' method the classifier is subject specific. For each subject, the epochs are partitioned into ten subsets, the classifier is trained on nine subsets and tested on one subset. Thereby the algorithm is validated ten times on each of the nine subjects (90-fold cross validation)

Total All epochs from all subjects were pooled, and partitioned into 20 subsets. A classifier was trained based on 19 sub-sets, and tested on the last subset. Cross-validation was performed over all 20 combinations.

Individual Same as 'Total', but only done on data from a single subject, which was split into ten subsets. Thus, there were 90 different test sets.

The three validation schemes each provide a different perspective on the sleep staging performance and the applicability of the method.

'Individual' is thought as a model of the scenario in which users have personal models/ classifiers created. This builds on an assumption that measurements from one night will have similar characteristics to those from a different night. This seems like a reasonable assumption, given the literature [22-24]. As shown in Fig. 2, test and training data was only picked from the same subject. Of course, as part of the calculation of the population Cohen's kappa value, all data was eventually used as test data (each test having its own training data).

In 'Leave-one-out', a pre-trained classifier was applied to data from a new subject, which is probably the most relevant scenario. However, in this study we only had nine subjects, which is likely much too low for any given subject to be well represented by the remainder of the population.

Therefore, we have included 'Total', which represents the scenario where the pool of subjects is very large, in which case all normal sleep phenotypes are assumed represented in the training data. In the limit of a very large subject group, it is expected that 'Leave-one-out' and 'Total' would converge, to a result in-between the results reported here. However, to achieve this would likely require a substantial number of subjects. 
During the analysis, we found that it is very important in 'Total' and 'Individual' that the test sets each form contiguous subsets of the data. If instead of the above, each subset was selected at random, it would mean that most likely each test epoch would have neighboring training epochs on both sides. This in turn would give the classifier access to the correct label for epochs extremely similar to the test epoch, preventing proper generalization, and leading to over fitting. We will return briefly to the discussion of this 'neighbor effect' later in the paper.

To evaluate the agreement between the expert labels and the output of the classifiers, Cohens kappa coefficient [25] was calculated for each of the three cross-validation methods.

\section{Results}

\section{Measurements}

All nine subjects managed to fall asleep wearing the PSG and ear-EEG equipment. One subject (number 5) reported having removed the right ear plug before falling asleep. When asked to judge their quality of sleep between the categories: unchanged-worsemuch worse, 1 subject reported "unchanged", 5 reported "worse" and 3 felt they slept much worse than usual. The subjects were not asked to describe whether their discomfort was caused by the ear-EEG device, the PSG, or both. The subjects slept (or attempted to sleep) between 2.4 and $9.6 \mathrm{~h}$ with the equipment on, an average of 6.9. This means that in total, $61.8 \mathrm{~h}$ of sleep were recorded and scored by the sleep scorer, resulting in 7411 30-s epochs. In Table 2 are shown the number of useable electrodes and scored epochs for each subject.

In the analysis below, the one-eared subject was not removed, instead all three derivations were identical for that subject.

\section{A first comparison}

Figure 3 shows characteristics of conventional EEG and ear-EEG, during sleep. Figure 3a shows power spectra for REM, NREM2 and NREM3 for two scalp derivatives and a left-right ear-EEG derivative. A large degree of similarity is observed for the scalp and ear derivatives, in particular REM and NREM spectra are clearly separated for all three derivatives. Figure $3 \mathrm{~b}$ shows characteristic sleep events (sleep spindle and K-complex) for the same two scalp derivatives and the left-right ear-EEG derivative. Clear similarities in the waveforms are observed across all three derivatives.

However, despite these similarities it cannot in general be assumed that sleep stage signatures will be exactly equal in conventional EEG and ear-EEG. Further, it should

Table $2 \kappa$ values for each subject, for all methods of cross-validation

\begin{tabular}{lllllllllll}
\hline Subject & $\mathbf{1}$ & $\mathbf{2}$ & $\mathbf{3}$ & $\mathbf{4}$ & $\mathbf{5}$ & $\mathbf{6}$ & $\mathbf{7}$ & $\mathbf{8}$ & $\mathbf{9}$ & Avr. \\
\hline Useable electrodes & 10 & 10 & 11 & 12 & 6 & 12 & 12 & 11 & 10 & 10.4 \\
Scored epochs & 1040 & 964 & 932 & 1150 & 491 & 926 & 758 & 293 & 857 & 823 \\
Leave-one-out, $\kappa$ & 0.05 & 0.36 & 0.57 & 0.60 & 0.03 & 0.59 & 0.75 & 0.65 & 0.44 & 0.45 \\
Total, $\kappa$ & 0.50 & 0.49 & 0.63 & 0.65 & 0.57 & 0.64 & 0.78 & 0.65 & 0.70 & 0.62 \\
Individual, $\kappa$ & 0.52 & 0.52 & 0.67 & 0.72 & 0.55 & 0.65 & 0.76 & 0.70 & 0.76 & 0.65
\end{tabular}

Averages were calculated as the average of all nine columns, not by weighting each subject by number of epochs 

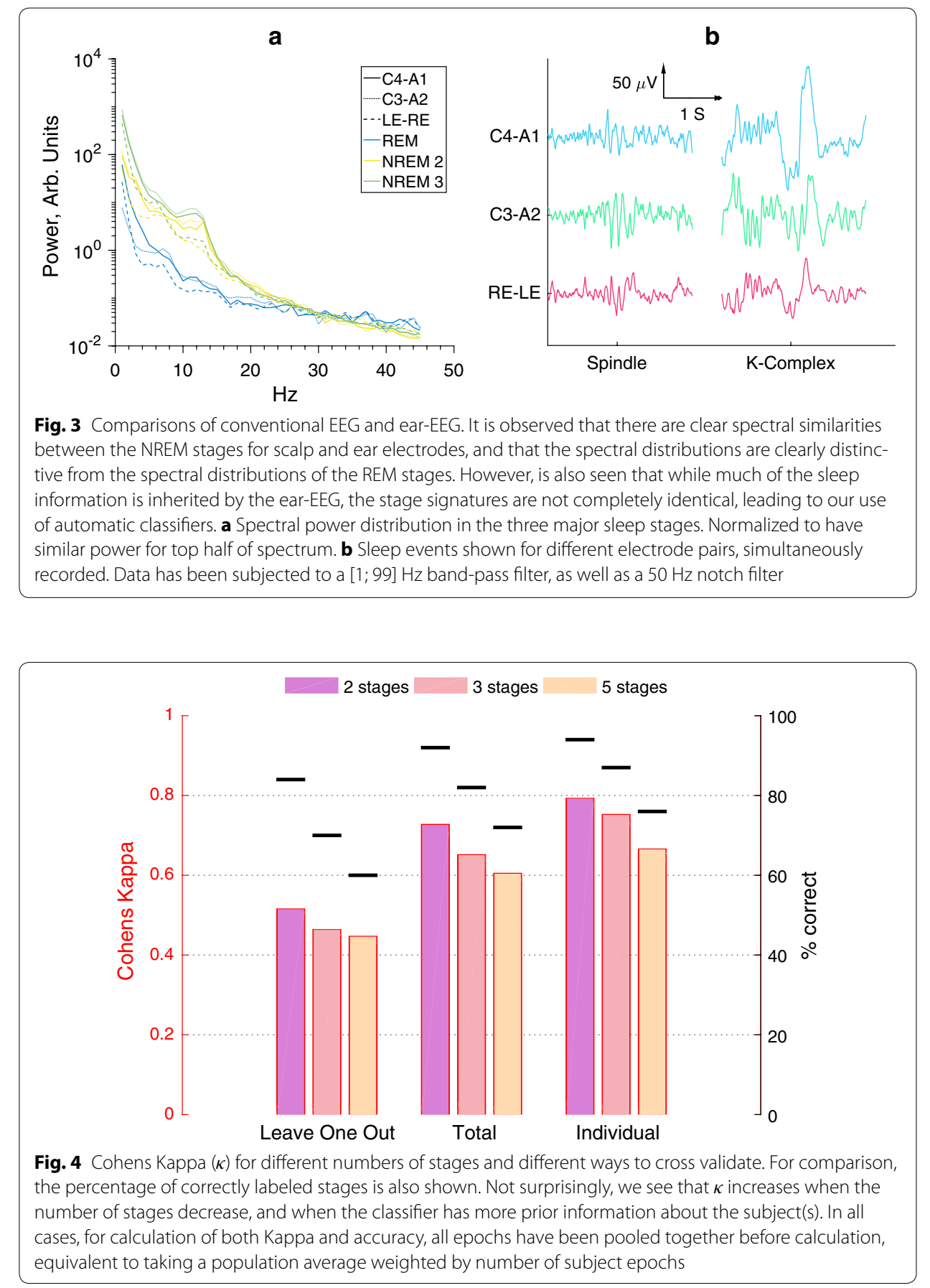

be stressed that not all sleep events are as clearly visible in ear-EEG as those shown. As was mentioned in the introduction, this is part of the reason why a machine learning approach is suitable for this study. More precisely, while we deem it likely that sleep experts could be trained, with some level of success, to score sleep based on ear-EEG data, it would likely require a significant amount of retraining, not suitable for this study. 

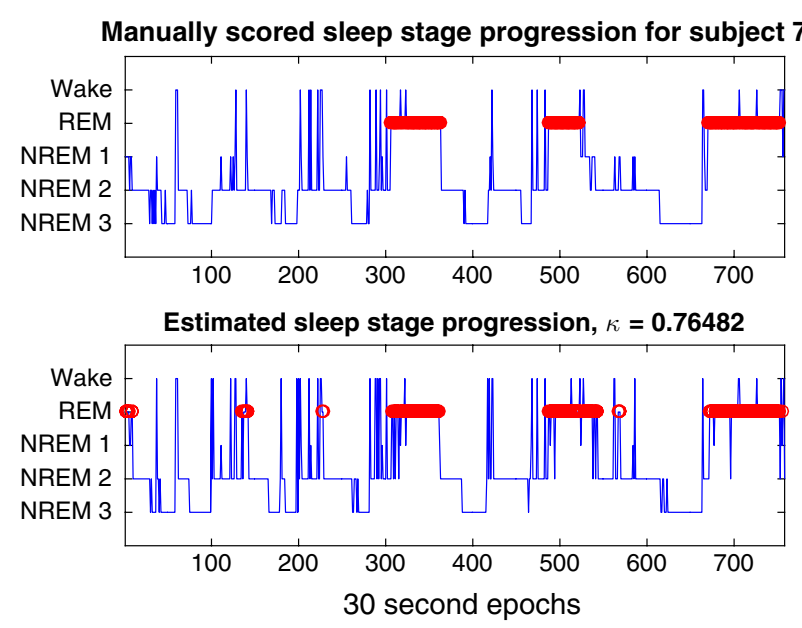

Fig. 5 Comparison of sleep staging results from manual (top) and automatic (bottom) staging, for one subject, using a classifier only trained on data from the same subject. REM stages have been highlighted in red, as per usual convention

\section{Classification results}

Figure 4 shows kappa values $(\kappa)$ for the three modes of cross validation and for 5, 3 and 2-stage classification (the stages in the last two being W-REM-NREM and W-Sleep, respectively). Results for 3 and 2-stage classification were simply obtained by relabelling the 5-stage results, so the classifiers were not retrained. Regarding the percentagewise agreement, it is noteworthy that manual scorers have been shown to have an average agreement of $82.6 \%$ [26], while actigraphs using 2 stages have an agreement rate of 83.996.5\% with PSG's [6].

For comparison, our classifier performs somewhat worse than the ones presented in [15] $(\kappa \approx 0.85)$ and [16] (correlation coefficient $\approx 0.84)$, though their studies did use scalp electrodes instead of ear-EEG.

When comparing the numbers shown in Fig. 4 to those found elsewhere in other studies, it is valuable to keep in mind that the 'neighbour effect' stemming from scattered test data, as was discussed above (see "Cross validation" section), may not always be accounted for in the literature. In our case, using scattered test data increased the percentagewise agreement between manual and automatic labels by an average $6 \%$ points across 'Total' and 'Individual'.

Figure 5 shows sleep staging traces for subject 7, using the 'Individual' cross-validation method. We see that generally the transitions between stable stages are accurately predicted.

Figure 6 shows the confusion matrices for the three cross validation schemes. The most difficult state to identify is NREM 1, likely stemming from the fact that there are very few examples of this (only 7\% of epochs). However, NREM 2 and NREM 3 are identified very well, even for 'Leave-one-out' cross validation.

In Table 2 is shown the $\kappa$-values for all subjects, for each method of cross validation. It is interesting to note that subject 5 was not always the worst performing subject, despite the fact that only data from one ear piece was available from this subject. 


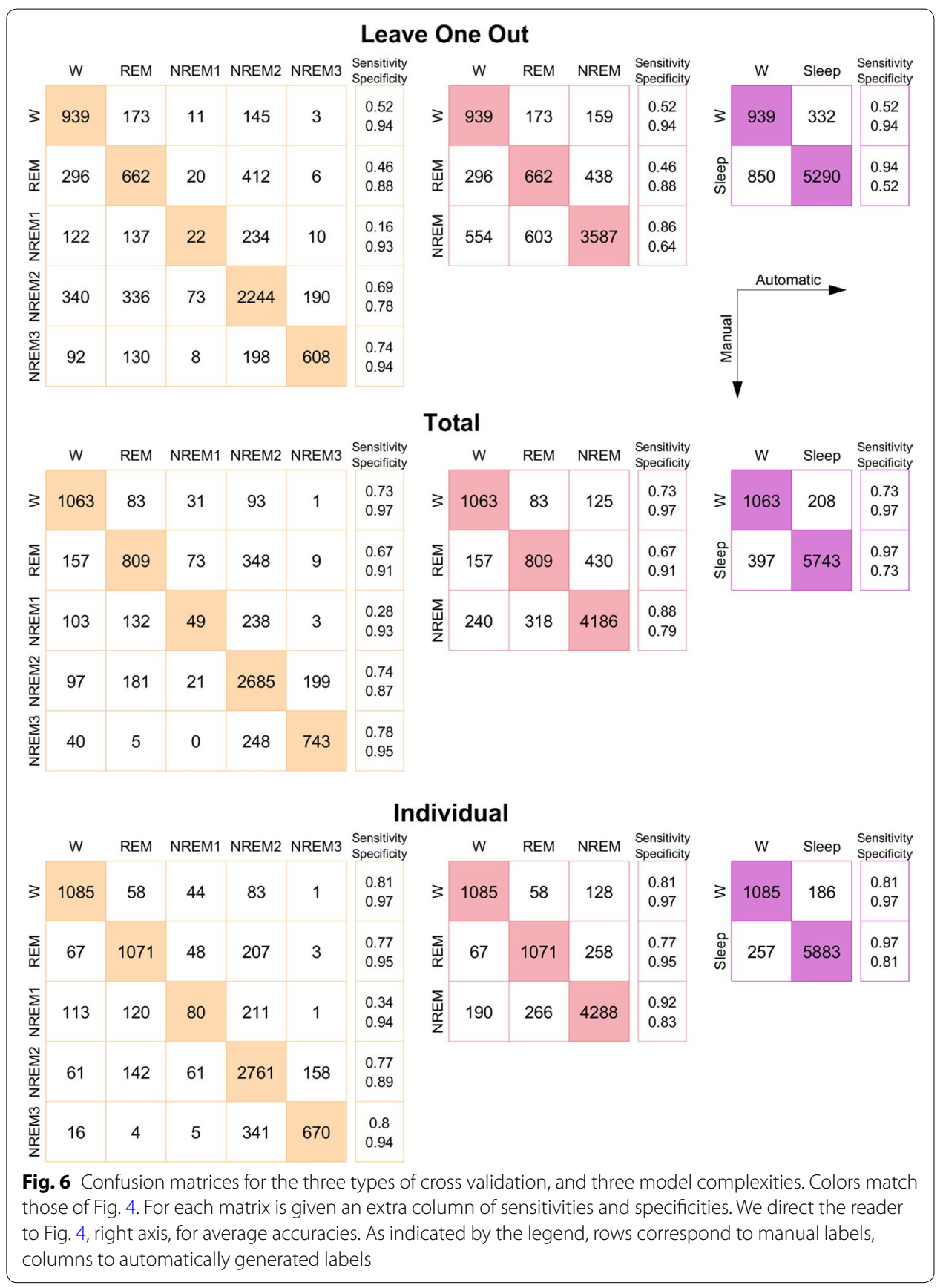

\section{Discussion}

We have seen that ear-EEG as a platform for automatic sleep staging has definite merit, especially if problems related to inter-subject variability can be addressed. Compared with other studies $[15,27,28]$, the subject cohort in this study is rather small at only nine individuals. However by resampling the cohort, it is possible to estimate the classifier performance for larger cohorts; following the procedure outlined in [29] we find that a cohort size of 30 would likely have increased the 5 -stage 'Leave-one-out' $-\kappa$ to 0.5 . 
An intriguing question which was not addressed here is intra-subject variability. In other words, how well does a classifier trained on data from Tuesday perform on data from Wednesday? It seems safe to say that it will at the very least be comparable to the 'Leave-one-out'-scheme described here, but possibly much closer to the 'Individual' scheme. Based on studies concerning individual differences in physiological measures during sleep [22-24], it seems likely that intra-subject variability will be low. In this scenario, one could imagine uses where a single night (possibly just a day-time nap) with both PSG and ear-EEG could be used to calibrate a classifier to each individual user. One example could be a clinical setting where the usual one night of PSG could be supplemented with a longer ear-EEG study spanning several weeks or more.

All data in this study was obtained from healthy individuals, and thus the study does not provide any information as to how ear-EEG would perform in the presence of pathology. However, given the demonstrated ability of ear-EEG to reliably classify sleep staging, it is likely that a specialist could utilize the technology to detect abnormal sleep.

A surprising issue during the study was that of user comfort. As soon as user discomfort was reported, a parallel investigation was initiated into possible remedies. These will be applied in a future study, where we expect the level of discomfort to be substantially reduced.

An additional benefit of the ear-EEG platform is the ease with which the electrodes remain attached to the skin. Whereas conventional electrodes need adhesives and/or mechanical support to ensure a reliable contact, ear-EEG benefits from the precise fit of the ear piece within the outer ear, largely retaining the connection through geometry alone.

\section{Conclusions}

The study makes the valuable contribution of having more participants than previous ear-EEG sleep studies, as well as being the first study to make a quantitative comparison to simultaneously recorded PSG.

Through the machine learning approach, the study amply demonstrates that ear-EEG contains sleep-relevant data, in line with previously published studies. However, the need for a comfortable sleep-monitoring solution is also highlighted. We are convinced, based on developments taking place after this study was conducted, that the comfort problems discussed here will be solved in future studies.

In summary, we consider the findings of this study very positive regarding the continued development of ear-EEG as a mobile sleep staging platform.

Sleep monitoring with ear-EEG will be particularly interesting in cases where it is relevant to monitor sleep over extended periods of time. In such cases automatic sleep staging turns out to be even more important and is probably a necessity. The findings in this study are also very positive in this regard.

In future studies, it would be interesting to add additional ways to compare measurements, for instance one in which the training and test sets were matched according to age and gender. This would most likely require a substantially larger pool of subjects. 
Authors' contributions

KBM was responsible for writing the manuscript. KBM, DBV and PK were responsible for data analysis and algorithmic development. DBV and $\mathrm{MO}$ were responsible for planning and carrying out the measurements and clinical scoring. PK was responsible for overall planning of the study. All authors read and approved the final manuscript.

\section{Author details}

${ }^{1}$ Department of Engineering, Aarhus University, Finlandsgade 22, 8200 Aarhus N, Denmark. ${ }^{2}$ Department of Clinical Medicine, Aarhus University, Nørrebrogade 44, 8000 Aarhus C, Denmark.

\section{Acknowledgements}

Not applicable.

Competing interests

The authors declare that they have no competing interests.

Availability of data and materials

All sleep measurements described in this paper are available for download at URL: http://www.eareeg.org/SleepData_2017/sleep.zip6. Bad channels have been marked by 'NaN'-values. The code used to sleep score automatically is available from the corresponding author (KM) upon request.

\section{Consent for publication}

At the time of their initial briefing, all study participants were informed of the likelihood that the data would be part of a publication.

\section{Ethics approval and consent to participate}

All subjects gave informed consent, and were briefed both verbally and in written form before their ear impressions were taken, in accordance with the regulations of the local ethics committee (Committee on Health Research Ethics, Jutland Central Region).

\section{Funding}

The work presented here was funded by Project 1311-00009B of the Danish strategic research council, and 110-2013-1 of the Danish national advanced technology foundation.

\section{Appendix A: Channel rejection}

To further elaborate on the choice of electrode rejection criteria, Fig. 7 shows $m_{i}$ for all channels. In the plot, electrodes that were expected to be bad either due to lost connection during the recording (an ear plug removed, for instance) or due to poor results in the initial ASSR test, have been marked in red. We see that the simple rejection criteria employed finds almost all these electrodes, and we consider this method both a more reproducible, as well as more scientifically sound approach.

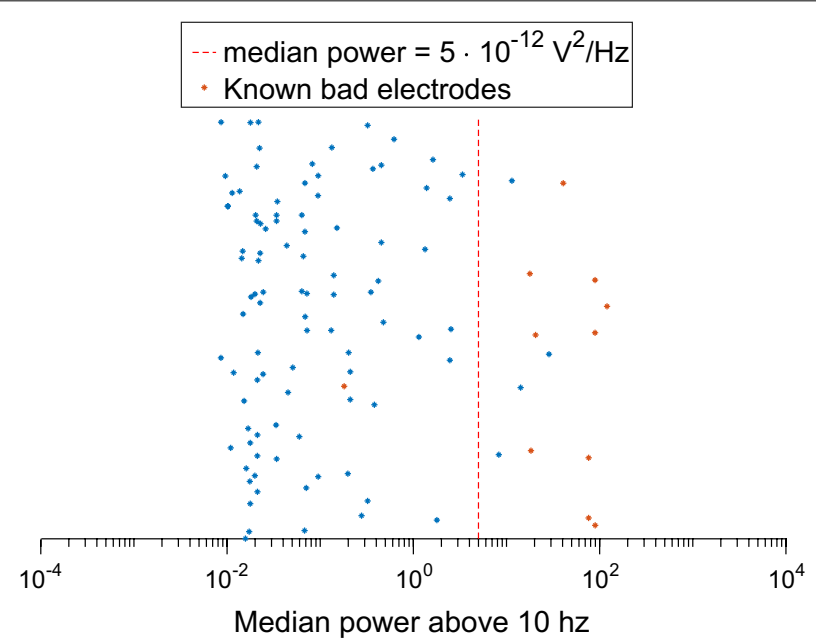

Fig. 7 The justification for the chosen threshold for electrode rejection. Each marker corresponds to an electrode, red markers show electrodes which, based on either ASSR measurements or visual inspection, were deemed unsuitable. We see that rejecting all electrodes above this limit corresponds quite well to the initial, more loosely defined criteria 


\section{Appendix B: Additional feature discussion}

\section{Feature elimination}

Feature elimination was attempted, by systematically removing one feature, and evaluating classifier performance, looping over all features. After each loop, the feature whose absence was the least detrimental to classifier performance was removed for the remainder of the analysis. In this way, the pool of features was gradually shrunk [30].

The data material in this test was all subjects pooled (called 'Total' above), and classifier performance was evaluated by partitioning the data pool into 20 equal parts, and iteratively training a classifier using $95 \%$ of the data as training data and the remaining $5 \%$ as test data. Finally, Cohen's $\kappa$ was calculated based on the combined classifier results.

Figure 8 shows the maximal $\kappa$ as a function of number of features. We see a general trend that best performance is achieved somewhere between 20 and 60 features. However, after further analysis, we have discovered that the precise set and number of features depends intimately not only on which subjects are included in the pool, but also how that pool is partitioned into 20 chunks. In other words, either the same, somewhat arbitrary choice of features is used, based on one chosen partitioning, in which case there is a risk of introducing a bias in the classifier (whichever representative subset of the data is chosen for selecting features, that subset may be overfitted when it is later used as test data), or there will be no clear indication of which set of features others should use. In the latter case, we would still have to present 99 features to our readers, and would have achieved no improvement in either classifier performance or readability. In future studies, we aim to have sufficient data to set aside a dedicated validation data set for determining feature selection, without the risk of overfitting. For now, we have chosen not to perform feature selection, but instead keep all 99 features.

\section{Feature details}

Below is given a detailed description of those features used which were not included in [15].

F7: Correlation coefficient between channels The only feature requiring multiple channels in its definition. Since there are three EEG derivations, this feature was simply cal-

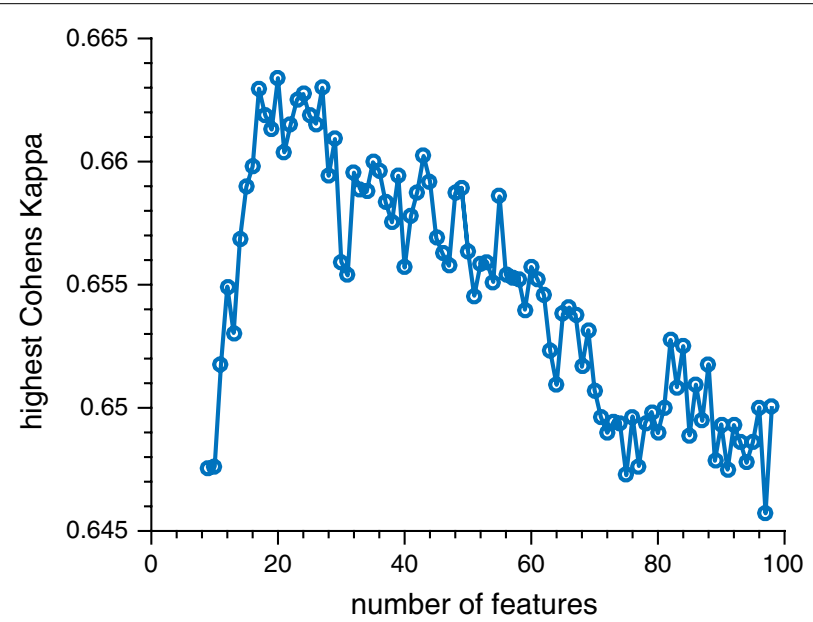

Fig. 8 Results from feature rejection. Features were gradually removed from the pool, in order to increase $\kappa$. At each step, the highest achieved value was recorded. Note the overall very low variation in $\kappa$ 
culated as a the correlation coefficient between the i'th and (i+1)'th derivations, making sure that all pairs of derivations are evaluated.

F8: EMG power Total power in the [32, 80] Hz band.

F9: Minimal EMG power Each epoch was split into ten segments. EMG power was calculated for each segment, and the lowest of these ten values was recorded.

F10: Relative EMG burst amplitude Maximum EMG signal amplitude divided by F9.

F11: Slow eye movement power Power in the frequency band $[0.5,2]$ relative to full power in the [0.5, 30] Hz-band. Inspired by Zhang et al. [31].

F12: Rapid eye movement power Power in the frequency band $[2,5]$ relative to full power in the [0.5, 30] Hz-band. Inspired by Zhang et al. [31].

F26: Mean spectral edge frequency difference Taken from [32].

F29: Spindle probability Letting $P(x-y)$ be the set of power estimates for frequencies in the $\mathrm{x}$ to $\mathrm{y} \mathrm{Hz}$ band, this feature is calculated as $\max (P(11-16)) /(\langle P(4-10)\rangle+\langle P(20-32)\rangle)$, and is inspired by Huupponen et al. [33] ("sigma index").

F30: Frequency stationarity For each epoch, the Welch algorithm calculates power spectra for 31 segments. F30 calculates the average Pearson correlation between these 31 spectra.

F31: Lowest adj. frequency similarity Using the same correlations as in F30, F31 is the lowest recorded correlation between neighboring segments.

F32: Largest CWT value A continuous Wavelet Transform of the filtered EEG-signal is computed, using a complex frequency B-spline as wavelet. The wavelet has a support of $0.5 \mathrm{~s}$. Inspired by Lajnef et al. [34].

F33: Longest sleep spindle The signal was bandpass filtered to a band of $11-16 \mathrm{~Hz}$, and the Teager energy operator (TEO) was applied to it (see [35]). At the same time, a short term Fourier transform (STFT) was applied to the unfiltered signal, and the power in the $12-14 \mathrm{~Hz}$ band relative to average power in $4-32 \mathrm{~Hz}$ band was computed (excluding $12-14 \mathrm{~Hz}$ ). Finally, signal segments in which F32 > 15, TEO > 0.5 and STFT power $>0.3$ were assumed to be sleep spindles. The maximal length of observed spindles constituted F33. This was inspired by Duman et al. [35].

As to the remaining features, the exact mapping is: (letting $\mathrm{KN}$ be the $\mathrm{N}$ 'th feature from [15]): F1:K3, F2:K4, F3: K5, F4: K7, F5: K8, F6:K9, F13:K14, F14:K15, F15:K16, F16:K17, F17:K18, F18:K19, F19:K20, F20:K21, F21:K22, F22:K23, F23:K24, F24:K25, F25:K27, F27:K26, F28: K28.

\section{Publisher's Note}

Springer Nature remains neutral with regard to jurisdictional claims in published maps and institutional affiliations.

Received: 11 May 2017 Accepted: 2 September 2017

Published online: 19 September 2017

\section{References}

1. Rechtschaffen A, Kales A. A manual of standardized terminology, techniques and scoring system for sleep stages of human subjects, No. 204. Washington, DC: National Institutes of Health publication; 1968.

2. Lamberg L. Promoting adequate sleep finds a place on the public health agenda. JAMA. 2004;291(20):2415. 
3. Taheri S. The link between short sleep duration and obesity: we should recommend more sleep to prevent obesity. Arch Dis Child. 2006;91(11):881-4.

4. Smaldone A, Honig JC, Byrne MW. Sleepless in America: inadequate sleep and relationships to health and wellbeing of our nation's children. Pediatrics. 2007;119(Supplement 1):29-37.

5. Stickgold R. Sleep-dependent memory consolidation. Nature. 2005;437(7063):1272-8.

6. Van de Water ATM, Holmes A, Hurley DA. Objective measurements of sleep for non-laboratory settings as alternatives to polysomnography—a systematic review. J Sleep Res. 2011;20(1 pt2):183-200.

7. Redmond SJ, de Chazal P, O'Brien C, Ryan S, McNicholas WT, Heneghan C. Sleep staging using cardiorespiratory signals. Somnologie-Schlafforschung und Schlafmedizin. 2007;11(4):245-56.

8. Kortelainen JM, Mendez MO, Bianchi AM, Matteucci M, Cerutti S. Sleep staging based on signals acquired through bed sensor. IEEE Trans Inf Technol Biomed. 2010;14(3):776-85.

9. Kidmose P, Looney D, Ungstrup M, Lind M, Mandic DP. A study of evoked potentials from ear-EEG. IEEE Trans Biomed Eng. 2013;60(10):2824-30.

10. Mikkelsen K, Kidmose P, Hansen LK. On the keyhole hypothesis: high mutual information between ear and scalp EEG neuroscience. Front Hum Neurosci. 2017;11:341. doi:10.3389/fnhum.2017.00341.

11. Zibrandtsen I, Kidmose P, Otto M, Ibsen J, Kjaer TW. Case comparison of sleep features from ear-EEG and scalp-EEG. Sleep Sci. 2016;9(2):69-72. doi:10.1016/j.slsci.2016.05.006.

12. Stochholm A, Mikkelsen K, Kidmose P. Automatic sleep stage classification using ear-EEG. In: 2016 38th annual international conference of the IEEE engineering in medicine and biology society (EMBC). New York: IEEE; 2016. p. 4751-4. doi:10.1109/embc.2016.7591789.

13. Looney D, Goverdovsky V, Rosenzweig I, Morrell MJ, Mandic DP. A wearable in-ear encephalography sensor for monitoring sleep: preliminary observations from Nap studies. Ann Am Thorac Soc. 2016.

14. Berry RB, Brooks R, Gamaldo CE, Hardsim SM, Lloyd RM, Marcus CL, Vaughn BV. The AASM manual for the scoring of sleep and associated events: rules, terminology and technical specifications, version 2.1. Darien: American Academy of Sleep Medicine; 2014

15. Koley B, Dey D. An ensemble system for automatic sleep stage classification using single channel EEG signal. Comput Biol Med. 2012;42(12):1186-95.

16. Doroshenkov L, Konyshev V, Selishchev S. Classification of human sleep stages based on EEG processing using hidden Markov models. Biomed Eng. 2007;41(1):25-8.

17. Acharya UR, Bhat S, Faust O, Adeli H, Chua EC-PC, Lim WJEJ, Koh JEWE. Nonlinear dynamics measures for automated EEG-based sleep stage detection. Eur Neurol. 2015;74(5-6):268-87.

18. Mikkelsen KB, Kappel SL, Mandic DP, Kidmose P. EEG recorded from the ear: characterizing the ear-EEG method. Front Neurosci. 2015:9:438. doi:10.3389/fnins.2015.00438.

19. Kidmose P, Looney D, Mandic DP. Auditory evoked responses from Ear-EEG recordings. In: Proc. of the 2012 annual international conference of the IEEE engineering in medicine and biology society (EMBC). New York: IEEE; 2012. p. 586-9. doi:10.1109/embc.2012.6345999.

20. Breiman L. Random forests. Mach Learn. 2001:45(1):5-32.

21. Ceriani L, Verme P. The origins of the Gini index: extracts from variabilità e mutabilità (1912) by Corrado Gini. J Econ Inequal. 2012;10(3):421-43.

22. Buckelmüller J, Landolt H-PP, Stassen HH, Achermann P. Trait-like individual differences in the human sleep electroencephalogram. Neuroscience. 2006;138(1):351-6.

23. Tucker AM, Dinges DF, Van Dongen HPA. Trait interindividual differences in the sleep physiology of healthy young adults. J Sleep Res. 2007;16(2):170-80.

24. Chua EC, Yeo SC, Lee IT, Tan LC, Lau P, Tan SS, Mien IH, Gooley JJ. Individual differences in physiologic measures are stable across repeated exposures to total sleep deprivation. Physiol Rep. 2014;2(9):12129.

25. McHugh ML. Interrater reliability: the kappa statistic. Biochem Med. 2012;22(3):276-82.

26. Rosenberg RS, Van Hout S. The American academy of sleep medicine inter-scorer reliability program: sleep stage scoring. J Clin Sleep Med. 2013;9(1):81-7.

27. Shambroom JR, Fábregas SE, Johnstone J. Validation of an automated wireless system to monitor sleep in healthy adults. J Sleep Res. 2012;21(2):221-30.

28. Stepnowsky C, Levendowski D, Popovic D, Ayappa I, Rapoport DM. Scoring accuracy of automated sleep staging from a bipolar electroocular recording compared to manual scoring by multiple raters. Sleep Med. 2013:14(11):1199-207.

29. Figueroa R, Treitler QZ, Kandula S, Ngo L. Predicting sample size required for classification performance. BMC Med Inform Decis Mak. 2012:12(1):8.

30. Guyon I, Elisseeff A. An introduction to variable and feature selection. J Mach Learn Res Spec Issue Var Feature Sel. 2003:3:1157-82

31. Zhang Y, Zhang X, Liu W, Luo Y, Yu E, Zou K, Liu X. Automatic sleep staging using multi-dimensional feature extraction and multi-kernel fuzzy support vector machine. J Healthc Eng. 2014;5(4):505-20.

32. Imtiaz S, Rodriguez-Villegas E. A low computational cost algorithm for REM sleep detection using single channel EEG. Ann Biomed Eng. 2014;42(11):2344-59.

33. Huupponen E, Gómez-Herrero G, Saastamoinen A, Värri A, Hasan J, Himanen S-LL. Development and comparison of four sleep spindle detection methods. Artif Intell Med. 2007;40(3):157-70.

34. Lajnef T, Chaibi S, Eichenlaub J-B, Ruby PM, Aguera P-E, Samet M, Kachouri A, Jerbi K. Sleep spindle and K-complex detection using tunable Q-factor wavelet transform and morphological component analysis. Front Hum Neurosci. 2015;9.

35. Duman F, Erdamar A, Erogul O, Telatar Z, Yetkin S. Efficient sleep spindle detection algorithm with decision tree. Expert Syst Appl. 2009:36(6):9980-5. 